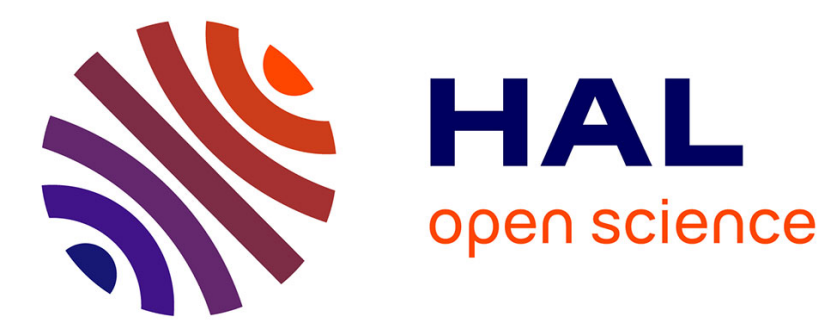

\title{
Aeroelastic instability analysis of NES-controlled systems via a mixed multiple scale/harmonic balance method
}

\author{
Angelo Luongo, Daniele Zulli
}

\section{- To cite this version:}

Angelo Luongo, Daniele Zulli. Aeroelastic instability analysis of NES-controlled systems via a mixed multiple scale/harmonic balance method. 2013. hal-00909812

\section{HAL Id: hal-00909812 \\ https://hal.science/hal-00909812}

Preprint submitted on 26 Nov 2013

HAL is a multi-disciplinary open access archive for the deposit and dissemination of scientific research documents, whether they are published or not. The documents may come from teaching and research institutions in France or abroad, or from public or private research centers.
L'archive ouverte pluridisciplinaire HAL, est destinée au dépôt et à la diffusion de documents scientifiques de niveau recherche, publiés ou non, émanant des établissements d'enseignement et de recherche français ou étrangers, des laboratoires publics ou privés. 


\title{
Aeroelastic instability analysis of NES-controlled systems via a mixed multiple scale/harmonic balance method
}

\author{
Angelo Luongo and Daniele Zulli
}

\begin{abstract}
The issue of passively controlling aeroelastic instability of general nonlinear multi-degree-of-freedom systems, suffering from Hopf bifurcation, is addressed. The passive device consists of an essentially nonlinear oscillator (nonlinear energy sink [NES]), having the task of absorbing energy from the main structure. The mathematical problem is attacked by a new algorithm, based on a suitable combination of the multiple scale and the harmonic balance methods. The procedure is able to furnish the reduced amplitude modulation equations, which govern the slow flow around a critical manifold, on which the equilibrium points lie. The method is applied to a sample structure already studied in literature, consisting of a two-degree-of-freedom rigid airfoil under steady wind. It is shown that NES, under suitable conditions, can shift forward the bifurcation point and, moreover, it can reduce the amplitude of the limit cycles. Relevant asymptotic results are compared, for validation purposes, with numerical simulations.
\end{abstract}

\section{Keywords}

Multiple scale method, harmonic balance, nonlinear energy sink, galloping, steady wind

\section{Introduction}

Nonlinear energy sinks (NESs) are constituted by essentially nonlinear oscillators, attached to a main structure. Their mass is generally small, compared with that of the main structure, and their goal is to absorb energy and to dissipate it, as a passive control device, through targeted energy transfers (TETs; see Vakakis et al. (2008a,b) for an extensive background). Their main feature, a consequence of the essential nonlinear nature, consists in the capacity, in principle, of being resonant at any frequencies, giving rise to large band tuning with the structure to be controlled.

The passive control of vibrations through the coupling of the main system with NESs has received a great deal of attention in the literature. In Gendelman et al. (2008) and Starosvetsky and Gendelman (2008b), a NES is applied to a main linear oscillator harmonically excited by a 1:1 resonant force. In Starosvetsky and Gendelman (2009), the same problem is extended to a NES with nonlinear damping. In Starosvetsky and Gendelman (2010b) a NES is applied to a free twodegree-of-freedom (2-d.o.f.) system in internal resonance, and in Starosvetsky and Gendelman (2008a) a harmonic external force is also considered. In Starosvetsky and Gendelman (2010a), for a harmonically forced linear (main) system, an extended approximation is suggested in order to analyze the strongly modulated responses (SMRs), typical for these systems, for growing values of the NES mass. The effects of a quasi-periodic, narrow-band random forcing on a linear oscillator equipped with a NES are studied in Starosvetsky and Gendelman (2011). In Vaurigaud, Savadkoohi and Lamarque (2011); Savadkoohi et al. (2012), multiple parallel NESs are considered to deal with a linear structure under impulse and harmonic forcing. In Pham et al. (2012) NES is used to control a linear oscillator under an external force with two different harmonics. In Musienko et al. (2006) the design of a NES constituted by a system of two oscillators,

DICEAA, University of L'Aquila, L'Aquila, Italy

Corresponding author:

Angelo Luongo, DICEAA, University of L'Aquila, via Giovanni Gronchi 18 , 67100 L'Aquila, Italy.

Email: angelo,luongo@univaq.it 
able to exploit nonlinear energy pumping, is carried out. In Lamarque et al. (2011) a nonsmooth NES has been introduced. In Vaurigaud, Manevitch and Lamarque (2011) a NES is used to control the flutter oscillations of a long-span bridge. In Vakakis et al. (2008b); Lee et al. (2007, 2008b,a); Gendelman et al. (2010), a NES is used to suppress aeroelastic instabilities on a class of rigid airfoils, modeled as a 2-d.o.f. section model, under stationary wind in the quasisteady hypothesis.

To study the slow-flow dynamics, the researchers very often make use of the complexification procedure by Manevitch (2001) and, in sequence, the multiple scale method (Nayfeh and Mook, 1979). In the same framework, in Gendelman (2004), the multiple scale method is applied, after complexification, by summing and subtracting a linear stiffness term in the NES equation, and properly ordering the dependent variables to avoid order violations.

On the other hand, in Luongo and Zulli (2012a) a new perturbation algorithm, based on a mixed multiple scale/harmonic balance method (MSHBM), is proposed to manage harmonically forced multi-d.o.f. dynamical systems. The main advantage of the algorithm is that the initial complexification procedure is avoided and the reducing process is re-conducted in the framework of the classical perturbation techniques. Moreover, whatever is the number of d.o.f. of the main system, the procedure directly provides the normal form equations (Nayfeh, 2011), i.e. the simplest reduced system describing the essential dynamics, constituted by a number of equations equal to the codimension of the system. In other words, the proposed technique applies the harmonic balance only to the NES equation, and it is done just because the solution of its essentially cubic equation is not expressed via simple functions, whereas the concurrent application of the MSM to the $n$-d.o.f. main system both filters the slow dynamics and reduces the system to the center manifold.

In this paper, inspired by Gendelman et al. (2010), a general, nonlinear, multi-d.o.f. aeroelastic system, close to a Hopf bifurcation which triggers flutter oscillations, is considered, and a NES is attached to it in order to control amplitude of vibrations. The mixed MSHBM (Luongo and Zulli, 2012a) is applied to get the critical manifold and the equations ruling the dynamics around it. The three reduced amplitude modulation equations (RAMEs) are obtained and discussed through the analysis of the equilibrium points and their stability. The validation of the method is pursued by a sample system, already analyzed by Gendelman et al. (2010), for which the asymptotic results are compared with numerical integrations of the original equations.
The paper is organized as follows: in Section 2, the algorithm is applied to a general aeroelastic system close to a Hopf bifurcation and the relevant equations are obtained; in Section 3 the sample system is studied and results discussed, and in Section 4 some conclusions are drawn.

\section{The multiple scale/harmonic balance algorithm}

\section{I. Equations of motion}

A quite general class of nonlinear, multi-d.o.f. mechanical systems, close to a Hopf bifurcation caused by aerodynamic forces, is considered (see Figure 1). The aerodynamic forces, due to the stationary wind of (nondimensional) speed $\mu$ which blows orthogonally to the plane of the structure, are assumed to be described by the quasi-steady theory. The main system is equipped with an essentially nonlinear oscillator behaving as a NES, attached at a point. The relevant nondimensional equations of motion read

$$
\begin{gathered}
\mathbf{M} \ddot{\mathbf{x}}+\mathbf{C}(\mu) \dot{\mathbf{x}}+\mathbf{K}(\mu) \mathbf{x}+\xi\left(\mathbf{r}^{T} \dot{\mathbf{x}}-\dot{y}\right) \mathbf{r} \\
+\kappa\left(\mathbf{r}^{T} \mathbf{x}-y\right)^{3} \mathbf{r}+\mathbf{f}(\mathbf{x}, \mathbf{x}, \mathbf{x})=0 \\
m \ddot{y}-\xi\left(\mathbf{r}^{T} \dot{\mathbf{x}}-\dot{y}\right)-\kappa\left(\mathbf{r}^{T} \mathbf{x}-y\right)^{3}=0
\end{gathered}
$$

where: $\mathbf{x}(t)$ is the time-depending $N$-dimensional vector of the displacements of the main structure; $\mathbf{M}$ is the mass matrix; $\mathbf{C}(\mu)$ is the (nonproportional) damping matrix and $\mathbf{K}(\mu)$ is the stiffness matrix, both including structural and aerodynamic effects, the latter depending on $\mu$, acting as a bifurcation parameter; $\mathbf{f}$ is the vector of the (cubic) geometric nonlinearity, assumed much

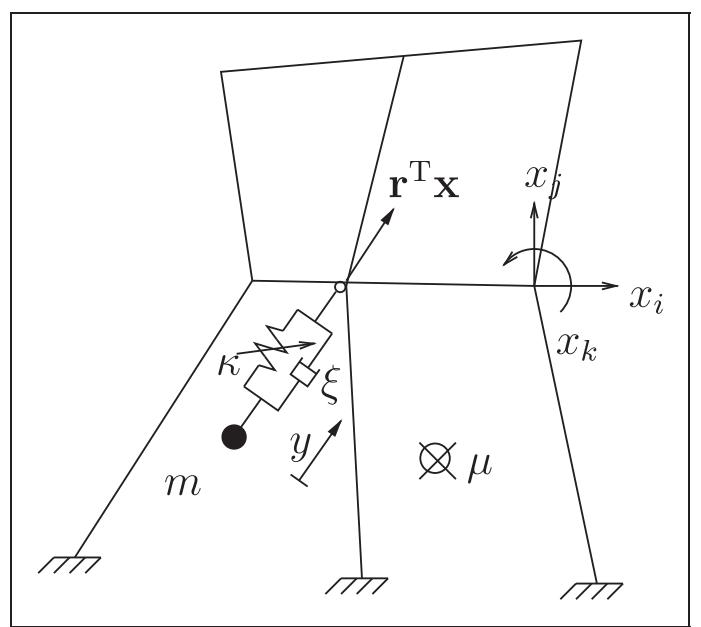

Figure I. Sketch of the aeroelastic multi d.o.f. system equipped with a NES. 
larger than the (neglected) aerodynamic nonlinearity, $y(t)$ is the time-dependent displacement of the added oscillator, $m$ its (small) mass, $\xi$ its (small) damping ratio and $\kappa$ the coefficient of its essentially nonlinear (cubic) spring; $\mathbf{r}$ is the influence coefficient vector; finally, the dot represents time-differentiation.

It is convenient to introduce the relative displacement between main structure and NES, $z:=\mathbf{r}^{T} \mathbf{x}-y$, so that Equations (1) become

$$
\begin{aligned}
& \mathbf{M} \ddot{\mathbf{x}}+\mathbf{C}(\mu) \dot{\mathbf{x}}+\mathbf{K}(\mu) \mathbf{x}+\xi \dot{z} \mathbf{r}+\kappa z^{3} \mathbf{r}+\mathbf{f}(\mathbf{x}, \mathbf{x}, \mathbf{x})=0 \\
& m\left(\ddot{z}-\mathbf{r}^{T} \ddot{\mathbf{x}}\right)+\xi \dot{z}+\kappa z^{3}=0
\end{aligned}
$$

It is interesting to note that, when $\xi=0$, the tangent operator of Equations (2) at $\mathbf{x}=\mathbf{0}$, admits a doublezero eigenvalue, due to the fact the NES has zero linear stiffness. The unfolding of this system is a family in which a small damping $\xi$ and a small linear stiffness $\kappa_{L}$ (here omitted) exist. Moreover, if the main structure manifests a Hopf bifurcation at a critical value $\mu=\mu_{0}$, a double-zero/Hopf bifurcation would occur at the point $\left(\mu_{0}, 0,0\right)$ of the parameter space $\left(\mu, \xi, \kappa_{L}\right)$, similarly to what studied in Luongo and Zulli (2012b). According to the center manifold theory (Guckenheimer and Holmes, 1983), such a circumstance would lead to the conclusion that, when $t \rightarrow \infty$, the motion develops itself on a four-dimensional manifold. However, such a bifurcation is not a standard one, since the mass of NES is evanescent, so that, in the limit for $m \rightarrow 0$, the two eigenvalues of the NES assume undetermined values. Such a pathological case, therefore, cannot be studied by the usual center manifold method, or by the multiple scale method, but a specific perturbation method must be tailored on it, as detailed below.

\subsection{MSHBM: first-order solution}

The dependent variables are first rescaled through a nondimensional small parameter $\epsilon>0$, as $(\mathbf{x}, z):=$ $\epsilon^{1 / 2}(\widetilde{\mathbf{x}}, \tilde{z})$, consistently with the presence of cubic nonlinearity, while the bifurcation parameter is expressed as $\mu=\mu_{0}+\epsilon \mu_{1}$, where $\mu_{0}$ is its critical value, to be still evaluated, and $\epsilon \mu_{1}$ is the small deviation from it. The parameters of the NES are also rescaled, since both the mass and the damping are assumed small: $(m, \xi):=$ $\epsilon(\tilde{m}, \tilde{\xi})$. The rescaling leads to the following equations, after omission of tilde and division by $\epsilon^{1 / 2}$ :

$$
\begin{aligned}
& \mathbf{M} \ddot{\mathbf{x}}+\left(\mathbf{C}_{0}+\epsilon \mu_{1} \mathbf{C}_{1}\right) \dot{\mathbf{x}}+\left(\mathbf{K}_{0}+\epsilon \mu_{1} \mathbf{K}_{1}\right) \mathbf{x}+\epsilon \xi \dot{z} \mathbf{r} \\
& \quad+\epsilon \kappa z^{3} \mathbf{r}+\epsilon \mathbf{f}(\mathbf{x}, \mathbf{x}, \mathbf{x})=0 \\
& \epsilon m\left(\ddot{z}-\mathbf{r}^{T} \ddot{\mathbf{x}}\right)+\epsilon \xi \dot{z}+\epsilon \kappa z^{3}=0
\end{aligned}
$$

where $\mathbf{C}_{0}:=\mathbf{C}\left(\mu_{0}\right), \mathbf{K}_{0}:=\mathbf{K}\left(\mu_{0}\right)$ and $\mathbf{C}_{1}:=\partial \mathbf{C}\left(\mu_{0}\right) / \partial \mu$, $\mathbf{K}_{1}:=\partial \mathbf{K}\left(\mu_{0}\right) / \partial \mu$

According to the multiple scale method, independent time scales $t_{0}:=t, t_{1}:=\epsilon t, \ldots$ are introduced and, consistently, the derivatives expressed as $\frac{\mathrm{d}}{\mathrm{d} t}=d_{0}+\epsilon d_{1}+\cdots$ and $\frac{\mathrm{d}^{2}}{\mathrm{~d} t^{2}}=d_{0}^{2}+2 \epsilon d_{0} d_{1}+\cdots$. Moreover, the dependent variables are expanded in series as

$$
\left\{\begin{array}{l}
\mathbf{x} \\
z
\end{array}\right\}=\left\{\begin{array}{l}
\mathbf{x}_{0} \\
z_{0}
\end{array}\right\}+\epsilon\left\{\begin{array}{l}
\mathbf{x}_{1} \\
z_{1}
\end{array}\right\}+\epsilon^{2}\left\{\begin{array}{l}
\mathbf{x}_{2} \\
z_{2}
\end{array}\right\}+\cdots
$$

Substituting in Equations (3) and collecting terms of the same order in $\epsilon$, leads to the following perturbation equations:

order $\epsilon^{0}$ :

$$
\mathbf{M} d_{0}^{2} \mathbf{x}_{0}+\mathbf{C}_{0} d_{0} \mathbf{x}_{0}+\mathbf{K}_{0} \mathbf{x}_{0}=0
$$

order $\epsilon^{1}$ :

$$
\begin{aligned}
& \mathbf{M} d_{0}^{2} \mathbf{x}_{1}+\mathbf{C}_{0} d_{0} \mathbf{x}_{1}+\mathbf{K}_{0} \mathbf{x}_{1} \\
& \quad=-2 \mathbf{M} d_{0} d_{1} \mathbf{x}_{0}-\mathbf{C}_{0} d_{1} \mathbf{x}_{0}-\mu_{1} \mathbf{C}_{1} d_{0} \mathbf{x}_{0}-\mu_{1} \mathbf{K}_{1} \mathbf{x}_{0} \\
& \quad-\xi d_{0} z_{0} \mathbf{r}-\kappa z_{0}^{3} \mathbf{r}-\mathbf{f}\left(\mathbf{x}_{0}, \mathbf{x}_{0}, \mathbf{x}_{0}\right) \\
& m d_{0}^{2} z_{0}+\xi d_{0} z_{0}+\kappa z_{0}^{3}=m \mathbf{r}^{T} d_{0}^{2} \mathbf{x}_{0}
\end{aligned}
$$

order $\epsilon^{2}$ :

$$
\begin{aligned}
& \mathbf{M} d_{0}^{2} \mathbf{x}_{2}+\mathbf{C}_{0} d_{0} \mathbf{x}_{2}+\mathbf{K}_{0} \mathbf{x}_{2}=-2 \mathbf{M} d_{0}\left(d_{2} \mathbf{x}_{0}+d_{1} \mathbf{x}_{1}\right)+\cdots \\
& m d_{0}^{2} z_{1}+\xi d_{0} z_{1}+3 \kappa z_{1} z_{0}^{2} \\
& \quad=-\xi d_{1} z_{0}-m\left[2 d_{0} d_{1} z_{0}-\mathbf{r}^{T}\left(d_{0}^{2} \mathbf{x}_{1}+2 d_{0} d_{1} \mathbf{x}_{0}\right)\right]
\end{aligned}
$$

It should be noted that, because of the vanishingly small values of the mass and damping, as well of the lack of linear stiffness, the equation of motion relevant to NES does not appear in the generator problem Equation (5 $)$, which therefore describes the linear dynamics of the main structure only, as if the NES is disengaged. It is assumed that, at the specific critical value $\mu_{0}$, the system experiences a Hopf bifurcation. This entails that the relevant eigenvalue problems

$$
\begin{aligned}
& \left(\lambda^{2} \mathbf{M}+\lambda \mathbf{C}_{0}+\mathbf{K}_{0}\right) \mathbf{u}=\mathbf{0} \\
& \left(\bar{\lambda}^{2} \mathbf{M}^{T}+\bar{\lambda} \mathbf{C}_{0}^{T}+\mathbf{K}_{0}^{T}\right) \mathbf{v}=\mathbf{0}
\end{aligned}
$$

have a solution $\lambda_{1,2}= \pm i \omega$, with the associated right ( $\mathbf{u}$ and $\overline{\mathbf{u}})$ and left ( $\mathbf{v}$ and $\overline{\mathbf{v}}$ ) eigenvectors (the overbar denoting the complex conjugate and $i$ the imaginary unit), whereas all the other eigenvalues have negative real parts and are far from the imaginary axis. Under these hypotheses, the steady solution of Equation $\left(5_{1}\right)$ is

$$
\mathbf{x}_{0}\left(t_{0}, t_{1}, \ldots\right)=A\left(t_{1}, \ldots\right) \mathbf{u} e^{i \omega t_{0}}+\text { c.c. }
$$

where $A\left(t_{1}, \ldots\right)$ is a complex amplitude whose modulation on the slower time scales must be evaluated, and c.c. stands for complex conjugate. 
The $\epsilon$-order perturbation equations $\left(5_{2,3}\right)$ are now addressed, and the NES equation considered first. Since its (steady) solution cannot be expressed by elementary (nor Jacobi) functions, the harmonic balance method is used, by letting

$$
z_{0}\left(t_{0}, t_{1}, \ldots\right)=\sum_{k} B_{0}^{(k)}\left(t_{1}, \ldots\right) e^{i k \omega t_{0}}+c . c .
$$

where $B_{0}^{(k)}\left(t_{1}, \ldots\right)$ are slowly modulated complex amplitudes to be evaluated. Equations (7) and (8) are substituted in Equation $\left(5_{3}\right)$ and $\omega$ frequency terms only balanced (other frequency terms, such as $3 \omega, 5 \omega, \ldots$, turn out to be of higher order, giving a negligible contribution to the dynamics of the system (Luongo and Zulli, 2012a)). This step leads to

$$
m \omega^{2}\left(-B_{0}+\mathrm{r}^{T} \mathbf{u} A\right)+i \xi \omega B_{0}+3 \kappa B_{0}^{2} \bar{B}_{0}=0
$$

where $B_{0}:=B_{0}^{(1)}$.

Equation (9) provides an algebraic constraint, through a co-dimension-two manifold, between the amplitude of oscillation of the main structure, $A$, and the amplitude of the NES elongation, $B_{0}$.

To get the polar form of Equation (9), $A:=\frac{1}{2} a e^{i \alpha}$ and $B_{0}:=\frac{1}{2} b e^{i \beta}$, are substituted in it and real and imaginary parts separated, thus obtaining

$$
\begin{aligned}
& -m \omega^{2}\left(b-\mathbf{r}^{T} \mathbf{u} a \cos \gamma\right)+\frac{3}{4} \kappa b^{3}=0 \\
& m \omega^{2} \mathbf{r}^{T} \mathbf{u} a \sin \gamma+\xi \omega b=0
\end{aligned}
$$

where $\gamma:=\alpha-\beta$ denotes the phase difference. Equations (10) can be manipulated to eliminate the phase-difference, to get

$$
\begin{gathered}
\frac{9}{16} \frac{\kappa^{2}}{\omega^{2}} b^{6}-\frac{3}{2} \kappa m b^{4}+\left(\xi^{2}+m^{2} \omega^{2}\right) b^{2} \\
-m^{2} \omega^{2}\left(\mathbf{r}^{T} \mathbf{u}\right)^{2} a^{2}=0
\end{gathered}
$$

which can be easily solved in the form $a=a(b)$.

Equation $\left(5_{2}\right)$ is then addressed, in which $z_{0}$ and $\mathbf{x}_{0}$ are now known. By requiring that the resonant forcing term is orthogonal to the null space of the adjoint operator (solvability condition), the following equation must hold:

$$
\begin{aligned}
\mathbf{v}^{H} & {\left[\left(2 i \omega \mathbf{M u}+\mathbf{C}_{0} \mathbf{u}\right) d_{1} A+\left(i \omega \mathbf{C}_{1} \mathbf{u}+\mathbf{K}_{1} \mathbf{u}\right) \mu_{1} A\right.} \\
& \left.+i \omega \xi \mathbf{r} B_{0}+3 \kappa \mathbf{r} B_{0}^{2} \bar{B}_{0}+3 A^{2} \bar{A} \mathbf{f}(\overline{\mathbf{u}}, \mathbf{u}, \mathbf{u})\right]=0
\end{aligned}
$$

producing the following differential equation:

$$
c_{1} d_{1} A=\mu_{1} c_{2} A+\xi c_{3} B_{0}+c_{4} B_{0}^{2} \bar{B}_{0}+c_{5} A^{2} \bar{A}
$$

where the expression of the complex coefficients $c_{i}$ is given in Appendix A. It is worth noting that, when $B_{0}=0$ is put into Equation (13), this reduces to the normal form equation for the Hopf bifurcation of the main system. This entails that the NES modifies both the bifurcation point and the limit cycle, thus bringing potential benefits to the mechanical behavior of the original system.

If one decided to stop the perturbation analysis at this step, Equations (13) should be joined to Equations (9). In this case, since the NES provides an algebraic constraint, its (complex) amplitude $B_{0}$ would be a passive variable, whereas the dynamic evolution of the (active) amplitude $A$ of the main system would be completely restrained onto the manifold Equation (11). Moreover, if the manifold exhibited turning points (see Figure 3 later), the motion could not cross them, since $\dot{A}$ does not vanish there, so that the equations would break down at singular points. To overcome this tight limitation, a further perturbation step must be accomplished.

\subsection{MSHBM: second-order solution}

The particular nondiverging solution of Equation $\left(5_{2}\right)$ is evaluated, taking into account the solvability condition Equation (13). In particular, substitution of Equation (13) in Equation $\left(5_{2}\right)$ leads to

$$
\begin{aligned}
\mathbf{M} & d_{0}^{2} \mathbf{x}_{1}+\mathbf{C}_{0} d_{0} \mathbf{x}_{1}+\mathbf{K}_{0} \mathbf{x}_{1} \\
= & -i \omega \mu_{1}\left[\mathbf{C}_{1} \mathbf{u}-\frac{1}{c_{1}}\left(\mathbf{v}^{H} \mathbf{C}_{1} \mathbf{u}\right)\left(2 i \omega \mathbf{M u}+\mathbf{C}_{0} \mathbf{u}\right)\right] A e^{i \omega t_{0}} \\
& -\mu_{1}\left[\mathbf{K}_{1} \mathbf{u}-\frac{1}{c_{1}}\left(\mathbf{v}^{H} \mathbf{K}_{1} \mathbf{u}\right)\left(2 i \omega \mathbf{M u}+\mathbf{C}_{0} \mathbf{u}\right)\right] A e^{i \omega t_{0}} \\
& -i \xi \omega\left[\mathbf{r}-\frac{1}{c_{1}}\left(\mathbf{v}^{H} \mathbf{r}\right)\left(2 i \omega \mathbf{M u}+\mathbf{C}_{0} \mathbf{u}\right)\right] B_{0} e^{i \omega t_{0}} \\
& -3 \kappa\left[\mathbf{r}-\frac{1}{c_{1}}\left(\mathbf{v}^{H} \mathbf{r}\right)\left(2 i \omega \mathbf{M u}+\mathbf{C}_{0} \mathbf{u}\right)\right] B_{0}^{2} \bar{B}_{0} e^{i \omega t_{0}} \\
& -3\left[\mathbf{f}(\overline{\mathbf{u}}, \mathbf{u}, \mathbf{u})-\frac{1}{c_{1}}\left(\mathbf{v}^{H} \mathbf{f}(\overline{\mathbf{u}}, \mathbf{u}, \mathbf{u})\right)\left(2 i \omega \mathbf{M u}+\mathbf{C}_{0} \mathbf{u}\right)\right] A^{2} \bar{A} e^{i \omega t_{0}} \\
& -\kappa \mathbf{r} B_{0}^{3} e^{3 i \omega t_{0}}-\mathbf{f}(\mathbf{u}, \mathbf{u}, \mathbf{u}) A^{3} e^{3 i \omega t_{0}}+c . c .
\end{aligned}
$$

The solution of Equation (14) is obtained through the superposition method, evaluating the solution related to any single addend of the right-hand side, and then summing them:

$$
\begin{aligned}
\mathbf{x}_{1}= & {\left[\left(i \omega \mathbf{w}_{1}+\mathbf{w}_{2}\right) \mu_{1} A+i \xi \omega \mathbf{w}_{3} B_{0}\right.} \\
& \left.+3 \kappa \mathbf{w}_{4} B_{0}^{2} \bar{B}_{0}+3 \mathbf{w}_{5} A^{2} \bar{A}\right] e^{i \omega t_{0}} \\
& +\left(\kappa \mathbf{w}_{6} B_{0}^{3}+\mathbf{w}_{7} A^{3}\right) e^{3 i \omega t_{0}}+\text { c.c. }
\end{aligned}
$$


where the expressions of the vectors $\mathbf{w}_{i}, i=1, \ldots, 7$ are given in Appendix A.

Equation $\left(5_{5}\right)$ is finally considered: a new harmonic balance is carried out, assuming the following expression for $z_{1}$ :

$$
z_{1}\left(t_{0}, t_{1}, \ldots\right)=\sum_{k} B_{1}^{(k)}\left(t_{1}, \ldots\right) e^{i k \omega t_{0}}+c . c .
$$

Substituting Equations (7), (8), (15) and (16) in Equation $\left(5_{5}\right)$ and balancing the $\omega$-frequency terms, the following equation is obtained $\left(B_{1}:=B_{1}^{(1)}\right)$ :

$$
\begin{aligned}
& m\left(-\omega^{2} B_{1}+2 i \omega d_{1} B_{0}+\omega^{2}\left[i \mu_{1} \omega \mathbf{r}^{T} \mathbf{w}_{1} A+\mu_{1} \mathbf{r}^{T} \mathbf{w}_{2} A\right.\right. \\
& \left.\quad+i \xi \omega \mathbf{r}^{T} \mathbf{w}_{3} B_{0}+3 \kappa \mathbf{r}^{T} \mathbf{w}_{4} B_{0}^{2} \bar{B}_{0}+3 \mathbf{r}^{T} \mathbf{w}_{5} A^{2} \bar{A}\right] \\
& \left.\quad-2 i \omega \mathbf{r}^{T} \mathbf{u} d_{1} A\right)+\xi d_{1} B_{0}+i \xi \omega B_{1} \\
& \quad+3 \kappa B_{0}^{2} \bar{B}_{1}+6 \kappa B_{0} \bar{B}_{0} B_{1}=0
\end{aligned}
$$

It appears that this equation describes the dynamics of the amplitude $B_{0}$, which becomes an active variable, differently from what is stated by Equation (9). The key term containing $d_{1} B_{0}$ comes out only at the second order, since it is affected by small coefficients $\xi$ and $m$, thus revealing the nature of singular perturbation.

To get the amplitude modulation equations (or bifurcation equations), Equations (9) and (17) must be combined with each other, and joined to the solvability condition (13). According to the usual reconstitution procedure, Equation (9) is multiplied by $\epsilon^{1 / 2}$, Equation (17) by $\epsilon^{3 / 2}$ and the two equations added; similarly, Equation (13) is multiplied by $\epsilon^{3 / 2}$. The true time $t$ appears in the process after that the substitution $\epsilon d_{1}=\mathrm{d} / \mathrm{d} t$ is performed; moreover, the NES total amplitude $B$, which collects the first- and second-order contributions, is introduced as $B:=B_{0}+\epsilon B_{1}$. Finally, the perturbation parameter $\epsilon$ is completely reabsorbed through the backward rescaling $\epsilon^{1 / 2} A \rightarrow A, \epsilon^{1 / 2} B \rightarrow$ $B, \epsilon m \rightarrow m, \epsilon \xi \rightarrow \xi, \epsilon \mu_{1} \rightarrow \mu_{1}$. However, in order to stress the nature of singular perturbation equation, $\epsilon$ is here left as a bookkeeping parameter in front of second-order terms (formally, the common factor $\epsilon^{3 / 2}$ is canceled). Hence, the reconstituted equations read

$$
\begin{aligned}
& c_{1} \dot{A}= \mu_{1} c_{2} A+\xi c_{3} B+c_{4} B^{2} \bar{B}+c_{5} A^{2} \bar{A} \\
& \epsilon\left[-2 i \omega m \mathbf{r}^{T} \mathbf{u} \dot{A}+(\xi+2 i \omega m) \dot{B}\right] \\
&=-m \omega^{2} \mathbf{r}^{T} \mathbf{u} A+\left(m \omega^{2}-i \xi \omega\right) B-3 \kappa B^{2} \bar{B} \\
& \quad-\epsilon m \omega^{2}\left[i \mu_{1} \omega \mathbf{r}^{T} \mathbf{w}_{1} A+\mu_{1} \mathbf{r}^{T} \mathbf{w}_{2} A\right. \\
&\left.\quad+i \xi \omega \mathbf{r}^{T} \mathbf{w}_{3} B+3 \kappa \mathbf{r}^{T} \mathbf{w}_{4} B^{2} \bar{B}+3 \mathbf{r}^{T} \mathbf{w}_{5} A^{2} \bar{A}\right]
\end{aligned}
$$

In polar coordinates, using the transformations $A(t):=\frac{1}{2} a(t) e^{i \alpha(t)}$ and $B(t):=\frac{1}{2} b(t) e^{i \beta(t)}$, and separating real and imaginary parts, four real equations are obtained, in the dependent variables $a, \alpha, b, \beta$. The definition of the phase difference $\gamma:=\alpha-\beta$ and consistent combination of the two phase equations, leads to three Reduced Amplitude Modulation Equations, in the dependent variables $a, b, \gamma$ :

$$
\begin{aligned}
\dot{a} & =\mathcal{F}\left(a, b, \gamma ; \mu_{1}\right) \\
\epsilon \dot{b} & =\mathcal{G}\left(a, b, \gamma ; \mu_{1}\right)=\mathcal{G}_{0}(a, b, \gamma)+\epsilon \mathcal{G}_{1}\left(a, b, \gamma ; \mu_{1}\right) \\
\epsilon a b \dot{\gamma} & =\mathcal{H}\left(a, b, \gamma ; \mu_{1}\right)=\mathcal{H}_{0}(a, b, \gamma)+\epsilon \mathcal{H}_{1}\left(a, b, \gamma ; \mu_{1}\right)
\end{aligned}
$$

where $\mathcal{F}, \mathcal{G}, \mathcal{H}$ are defined in Appendix A. The fourth remaining equation turns out to be useful just to evaluate the value of $\alpha$ or $\beta$ once known $\gamma$.

\subsection{Critical manifold and steady solutions}

Equations (19) have the classical form of singular perturbation equations, since a small parameter (essentially denoting the smallness of the NES mass and damping) affects the first derivative of some slow-fast state variables ( $b$ and $\gamma$ in our case). Such equations, as is well known, produce a slow dynamics around a critical manifold $\mathcal{M}$ of the state space, and a fast dynamics far from $\mathcal{M}$ (Guckenheimer et al., 2006). The critical manifold is defined as the locus of the zero-velocity points of the slow-fast variables, namely,

$$
\begin{aligned}
\mathcal{G}\left(a, b, \gamma ; \mu_{1}\right) & =0 \\
\mathcal{H}\left(a, b, \gamma ; \mu_{1}\right) & =0
\end{aligned}
$$

For a fixed value of the wind velocity $\mu_{1}$, Equations (20) describe a curve in a 3D space (see Figure 6 later for an example); if $\mu_{1}$ is allowed to vary, a family of critical manifolds exists, parametrized by $\mu_{1}$.

When the state is close to the manifold, i.e. $\mathcal{G}=\mathcal{O}(\epsilon), \mathcal{H}=\mathcal{O}(\epsilon)$, then $\dot{b}=\mathcal{O}(1)$ and $\dot{\gamma}=\mathcal{O}(1)$; since $\dot{a} \leq \mathcal{O}(1)$ everywhere, the flow is slow. When the state is far from the manifold, i.e. $\mathcal{G}=\mathcal{O}(1), \mathcal{H}=\mathcal{O}(1)$, then $\dot{b}=\mathcal{O}\left(\epsilon^{1}\right)$ and $\dot{\gamma}=\mathcal{O}\left(\epsilon^{1}\right)$, so that the flow is fast. Therefore, far from $\mathcal{M}$, the motion develops essentially in an $a=$ const. plane, and possibly tends to stable branches of $\mathcal{M}$. Close to $\mathcal{M}$, however, all of the velocities are $\mathcal{O}(1)$, so that the motion slowly develops itself in a thin tube of radius $\mathcal{O}(\epsilon)$ around $\mathcal{M}$. On the other hand, when folds of the manifold are approached (at which the tangent is parallel to the $(b, \gamma)$-plane), then the system leaves the tube and jumps fast to another stable branch. Relaxation oscillations are motions in which jumps and slow evolutions cyclically alternate.

An asymptotic expression for the manifold is searched in the following way. First, the variable $\gamma$ is 


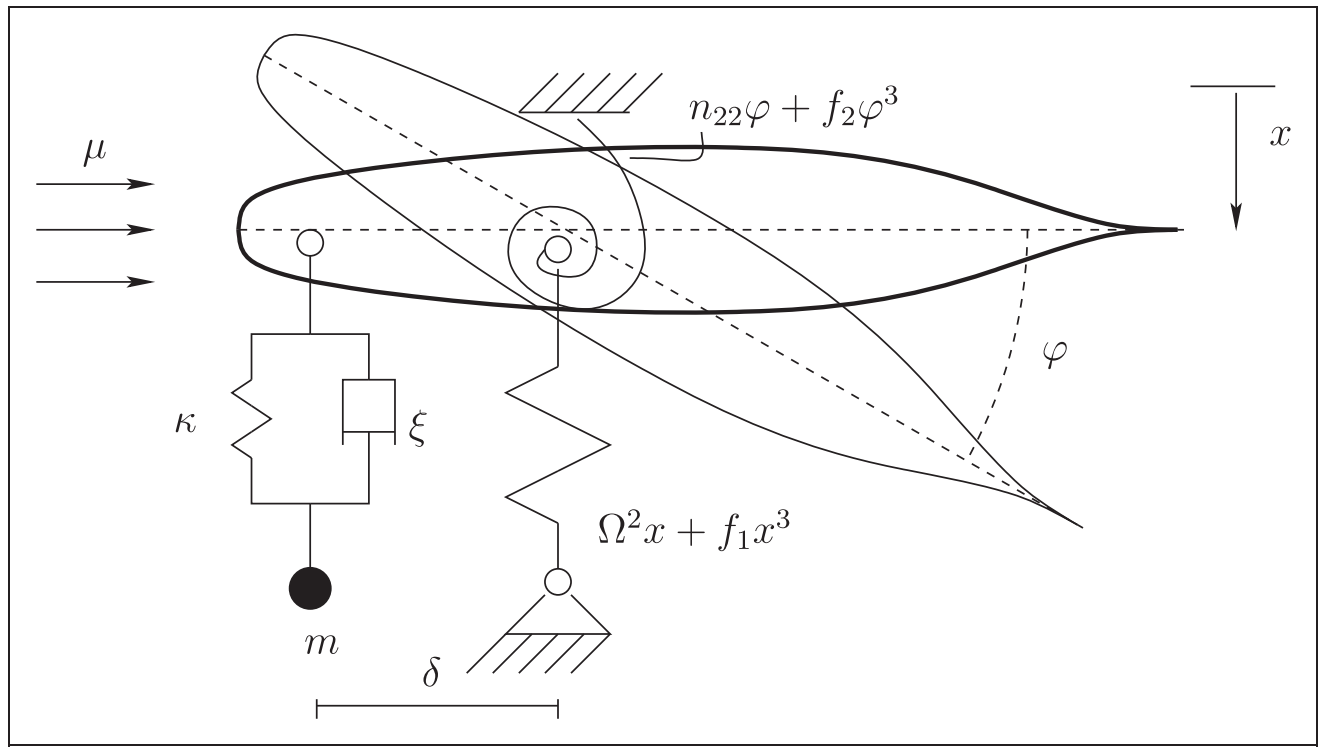

Figure 2. Rigid airfoil with NES under steady wind.

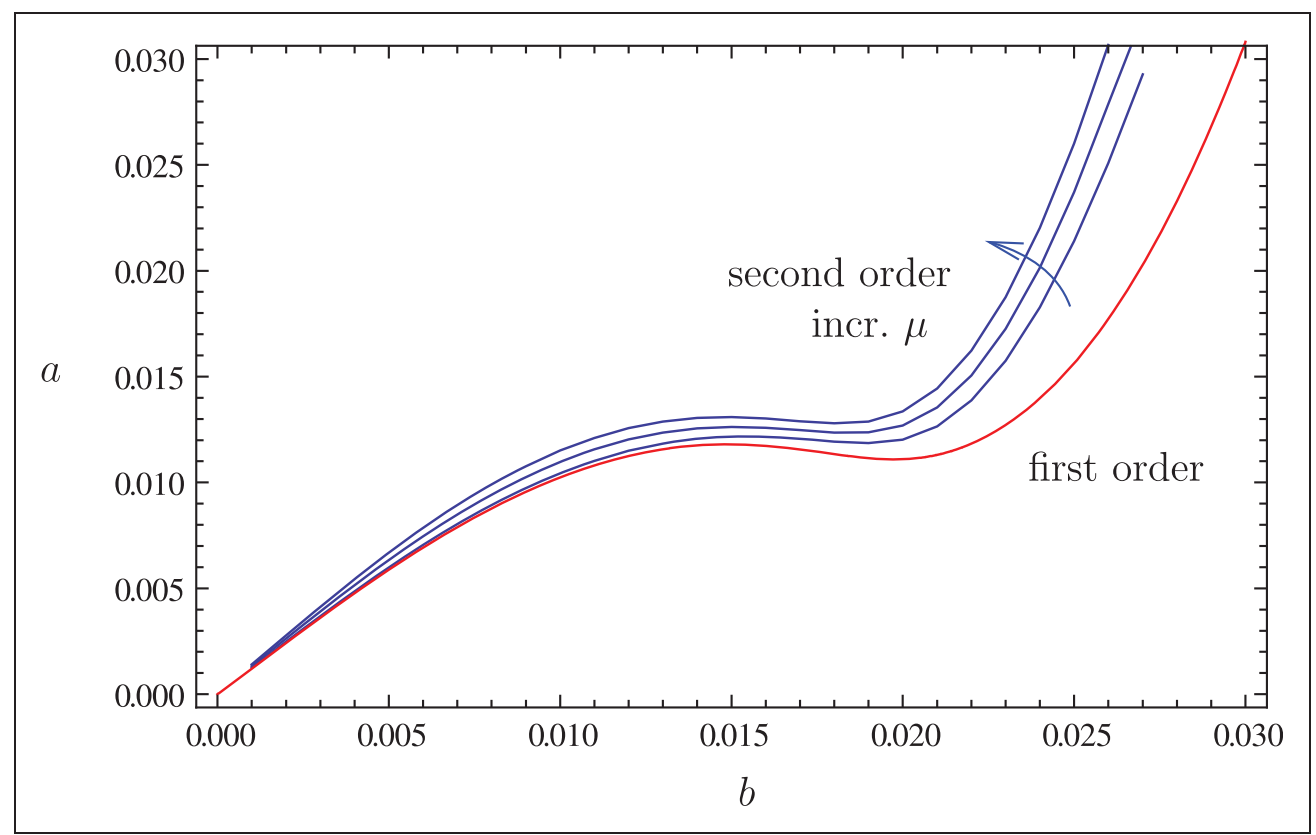

Figure 3. Representation of the manifold on the plane $(b, a)$, for $\kappa$ second order approximation for $\mu_{1} \quad(0 ; 0.05 ; 0.1)$.

40 and $\delta \quad$ 0.9. Red line: first order approximation; blue lines:

eliminated from the two Equations (20), this leading to a unique equation of type $\mathcal{M}_{0}(a, b)+\epsilon \mathcal{M}_{1}\left(a, b ; \mu_{1}\right)=0$. To solve this equation in the form $a=a\left(b ; \mu_{1}\right), a$ is expanded as $a=a_{0}+\epsilon a_{1}$ and two perturbation equations $\quad \mathcal{M}_{0}\left(a_{0}, b\right)=0 \quad$ and $\quad \mathcal{M}_{0, a}\left(a_{0}, b\right) a_{1}+$ $\mathcal{M}_{1}\left(a_{0}, b ; \mu_{1}\right)=0$ are derived, where a comma denotes differentiation with respect to the following argument. The first perturbation equation supplies the leading term $a_{0}(b)$ (which is independent of the wind velocity), the second equation furnishes the correction $a_{1}\left(b ; \mu_{1}\right)$ (which, in contrast, is wind-dependent). Thus, an asymptotic expression for the projection of the family of manifolds onto the $(a, b)$-plane is derived. If of interest, the phase difference $\gamma\left(a(b) ; \mu_{1}\right)$ can be successively evaluated. The expressions of $\mathcal{M}_{0}, \mathcal{M}_{1}$ are not shown for the sake of brevity.

The equilibrium points of system (19) are obtained by taking $\dot{a}=\dot{b}=\dot{\gamma}=0$. Therefore, they solve the 


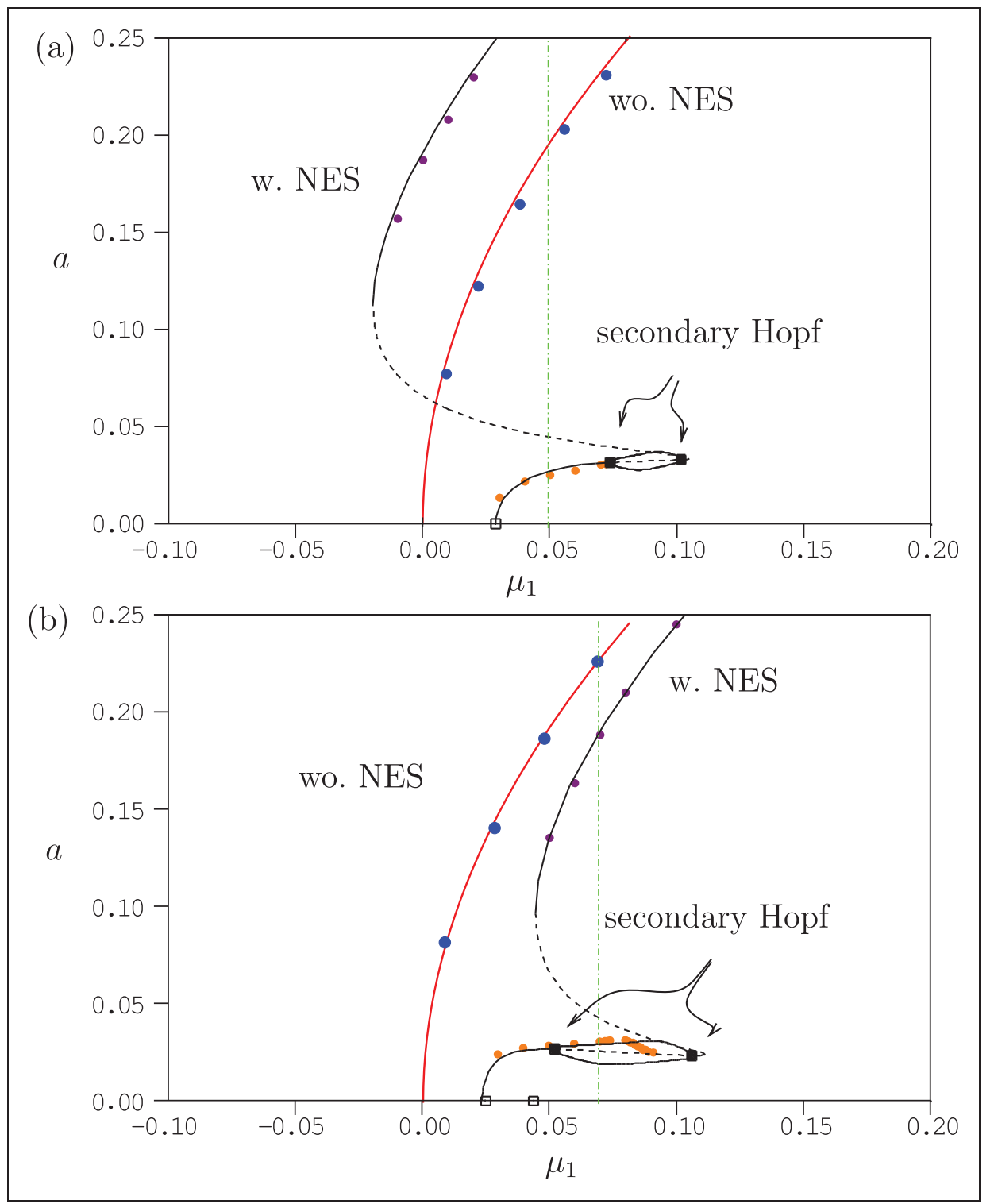

Figure 4. Equilibrium branches of the slow flow on the plane $\left(\mu_{1}, a\right):(a) \kappa \quad 10, \delta \quad 0.75 ;(b) \kappa \quad 10, \delta \quad 0.75$. Red line: without NES; black line: with NES; dots: numerical integrations of the originating equations; continuous line: stable; dashed line: unstable; black square: secondary Hopf point.

following nonlinear system:

$$
\begin{array}{r}
\mathcal{F}\left(a, b, \gamma(a, b) ; \mu_{1}\right)=0 \\
\mathcal{M}\left(a, b ; \mu_{1}\right)=0
\end{array}
$$

Once obtained the equilibrium points (involving both $a \neq 0$ and $b \neq 0$ ), their stability is determined by the sign of the real part of the eigenvalues of the Jacobian matrix $\mathbf{J}_{e}$ of Equations (19), which rules the linearized system around them

$$
\left\{\begin{array}{l}
\delta \dot{a} \\
\delta \dot{b} \\
\delta \dot{\gamma}
\end{array}\right\}=\mathbf{J}_{e}\left\{\begin{array}{l}
\delta a \\
\delta b \\
\delta \gamma
\end{array}\right\}
$$

\section{Numerical results}

A sample system, already considered by Gendelman et al. (2010), is used to evaluate the reliability of the MSHBM. It is constituted by a 2 d.o.f. rigid airfoil subjected to the (nondimensional) steady wind $\mu$, and is sketched in Figure 2. The (nondimensional) Lagrangian parameters are $x$ and $\varphi$, representing the plunge and the pitch, respectively. The two nonlinear springs, extensional and rotational respectively, have both linear and cubic coefficients. The position of the NES with respect to the center of mass of the airfoil is described by the (nondimensional) parameter $\delta$ : if it is positive, then the NES is windward; otherwise, if $\delta$ is negative, the NES is leeward. 


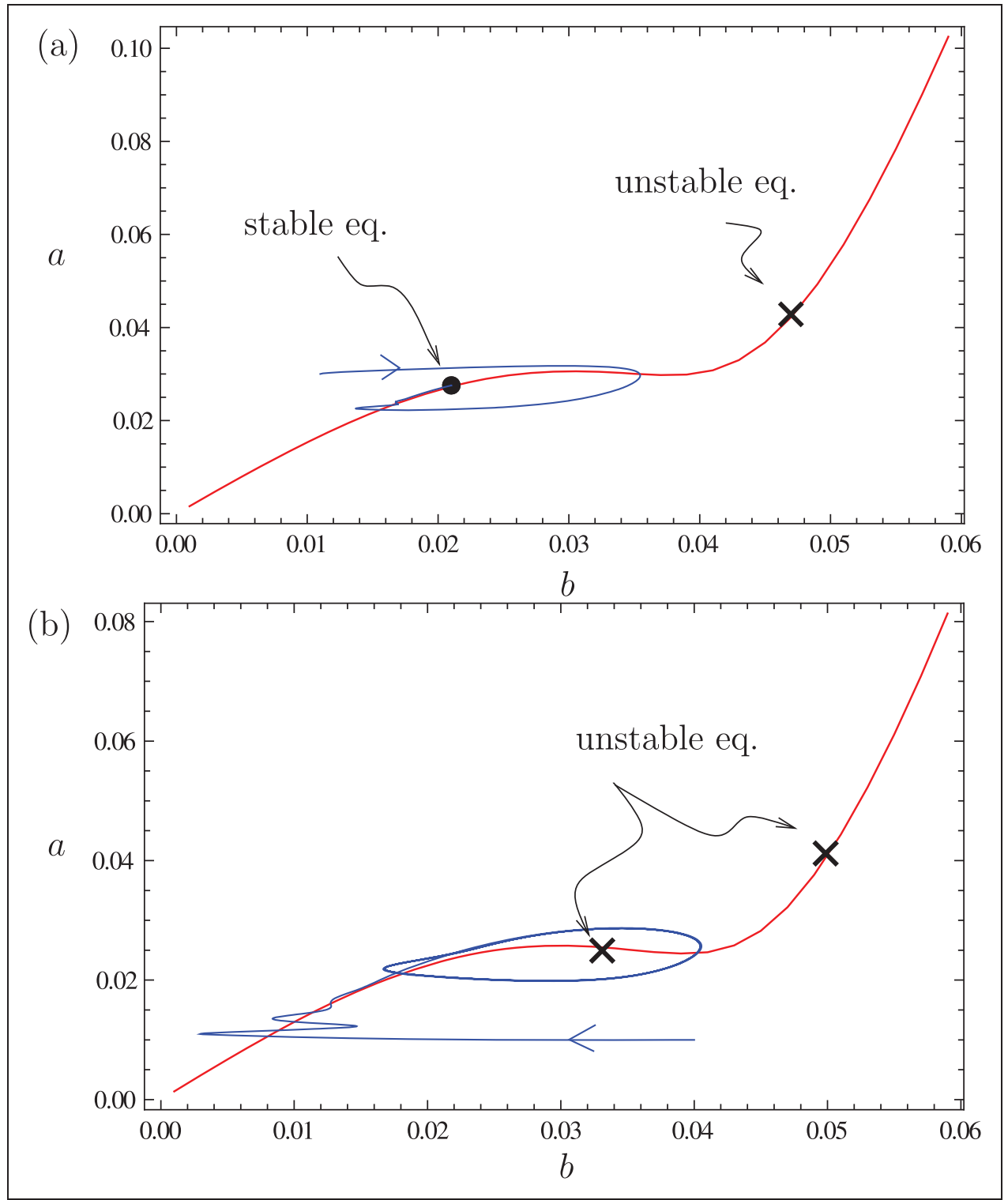

Figure 5. Phase portrait on the plane $(b, a):(a) \kappa \quad 10, \mu_{1} \quad 0.05, \delta \quad 0.75 ;(b) \kappa \quad 10, \mu_{1} \quad 0.07, \delta \quad 0.75$. Red line: manifold; blue line: trajectory; black circle: stable equilibrium point; black cross: unstable equilibrium point.

The nondimensional equations of motion are

$$
\begin{aligned}
& \ddot{x}+n_{12} \ddot{\varphi}+\mu g_{11} \dot{x}+\Omega^{2} x+\mu^{2} g_{11} \varphi-\xi(\dot{y}-\dot{x}+\dot{\alpha} \delta) \\
& \quad-\kappa(y-x+\alpha \delta)^{3}+f_{1} x^{3}=0 \\
& n_{12} \ddot{x}+n_{22} \ddot{\varphi}+\mu g_{21} \dot{\varphi}+k_{21} x+\left(n_{22}-\mu^{2} g_{21}\right) \varphi \\
& \quad+\xi(\dot{y}-\dot{x}+\dot{\alpha} \delta) \delta+\kappa(y-x+\alpha \delta)^{3} \delta+f_{2} \varphi^{3}=0 \\
& m \ddot{y}+\xi(\dot{y}-\dot{x}+\dot{\alpha} \delta)+\kappa(y-x+\alpha \delta)^{3}=0
\end{aligned}
$$

The comparison between Equations (23) and (1) allows one to identify the relevant matrices and columns as

$$
\begin{array}{r}
\mathbf{x}=\left\{\begin{array}{l}
x \\
\varphi
\end{array}\right\}, \quad \mathbf{M}=\left[\begin{array}{cc}
1 & n_{12} \\
n_{12} & n_{22}
\end{array}\right], \quad \mathbf{C}(\mu)=\mu\left[\begin{array}{ll}
g_{11} & 0 \\
g_{21} & 0
\end{array}\right], \\
\mathbf{K}(\mu)=\left[\begin{array}{cc}
\Omega^{2} & \mu^{2} g_{11} \\
k_{21} & n_{22}-\mu^{2} g_{21}
\end{array}\right], \quad \mathbf{r}=\left\{\begin{array}{c}
1 \\
-\delta
\end{array}\right\}, \quad \mathbf{f}=\left\{\begin{array}{l}
f_{1} x^{3} \\
f_{2} \varphi^{3}
\end{array}\right\}
\end{array}
$$

The following numerical values are chosen, corresponding to those used by Gendelman et al. (2010):

$$
\begin{aligned}
n_{12} & =n_{21}=0.2, n_{22}=0.25, g_{11}=0.2, g_{21}=-0.08, \\
\Omega & =0.5, k_{21}=0, f_{1}=f_{2}=1, m=0.02
\end{aligned}
$$




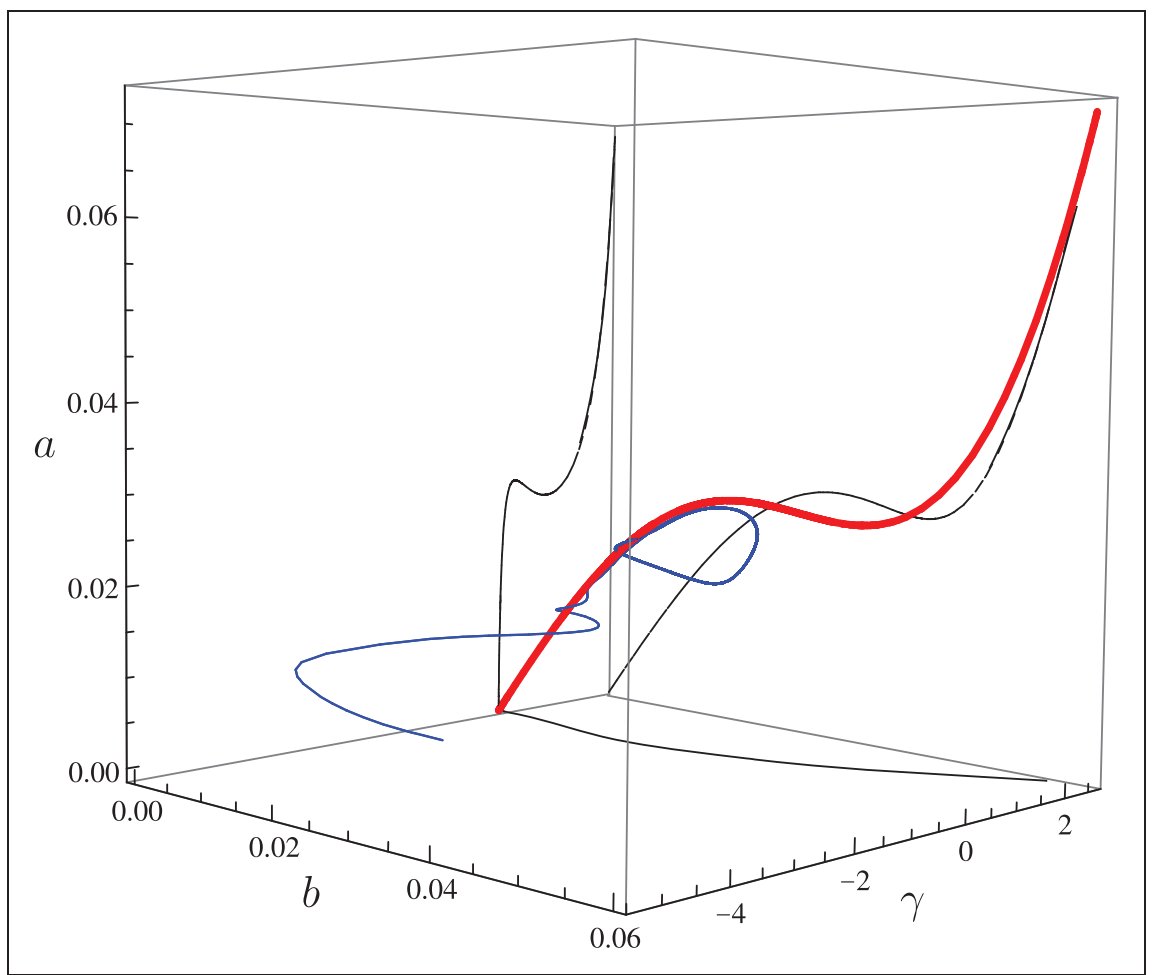

Figure 6. Phase portrait on the space $(b, \gamma, a): \kappa$ 10, $\mu_{1} \quad 0.07$ and $\delta$ 0.75. Red line: manifold; blue line: trajectory; black lines: projections on the coordinate planes.

and $\xi=0.008$. For the specified values, the critical wind turns out to be $\mu_{0}=0.8704$, the corresponding critical frequency $\omega=0.8704$ (imaginary part of the eigenvalue) and the right and left eigenvectors $\mathbf{u}=\{0,1\}^{T}$ and $\quad \mathbf{v}=\{-0.6521-0.5635 i, 0.5217+2.7486 i\}^{T}$, respectively.

The manifold is shown in Figure 3, where the red line represents the first approximation $\mathcal{M}_{1}(a, b)=0$ whereas the blue lines correspond to the second-order approximation, for different values of $\mu_{1}$.

In Figure 4 the equilibrium branches of the RAMEs, corresponding to periodic motions in the variables $x, \varphi, z$, are shown for (a) windward NES $(\delta=0.75)$ and (b) leeward NES $(\delta=-0.75)$. The red line describes the branch when the NES is disengaged, and the dots represent results of the numerical integration of the original equations (23), which are in good agreement. It can be seen that, when the NES is disengaged, a supercritical Hopf bifurcation occurs at $\mu_{1}=0$ and stable periodic motions are triggered for increasing values of $\mu_{1}$, whose amplitudes are represented by the red line. The NES shifts forward the position of the bifurcation points, but it also makes the bifurcation sub-critical. Indeed two turning points occur, as well as two secondary Hopf points which trigger stable periodic motions in $a$, corresponding to quasi-periodic motions in $x, \varphi$ and $z$ (the amplitude of the limit cycles are shown in the pictures). In case of windward NES (Figure 4a), next to the second turning point, the amplitude of the branch is larger than that without NES. It means that, in this case, the NES gives a harmful contribution to the dynamics of the system. On the other hand, in case of leeward NES (Figure 4b), the branch of the amplitude is always underneath the one corresponding to the case without NES. Therefore, for leeward NES, the effective reduction of the amplitude of oscillations is accomplished. These results are in agreement with Vakakis et al. (2008b). The two vertical dashed-dotted green lines in Figure 4 represent the values of $\mu_{1}$ for which the phase portraits of Figure 5 are produced ( $\mu_{1}=0.05$ and $\mu_{1}=0.07$, respectively). In particular, from Figure 5(a) it is evident how the stable equilibrium point (black circle), which lies on the manifold, asymptotically attracts the dynamic evolution of the system; as a correspondence, periodic oscillations in the variables $(x, \varphi, z)$ are produced. On the other hand, in Figure 5(b), realized for a value of $\mu_{1}$ between the two secondary Hopf bifurcations, the equilibrium points are unstable, and a limit cycle in $(b, a)$ is obtained. The phase portrait is also shown in the 3D space $(b, \gamma, a)$ (Figure 6) where it is evident that, after a transient where the slow flow is attracted by the stable branch of the manifold, the limit cycle of the relaxation oscillations arises. It corresponds to quasi-periodic 


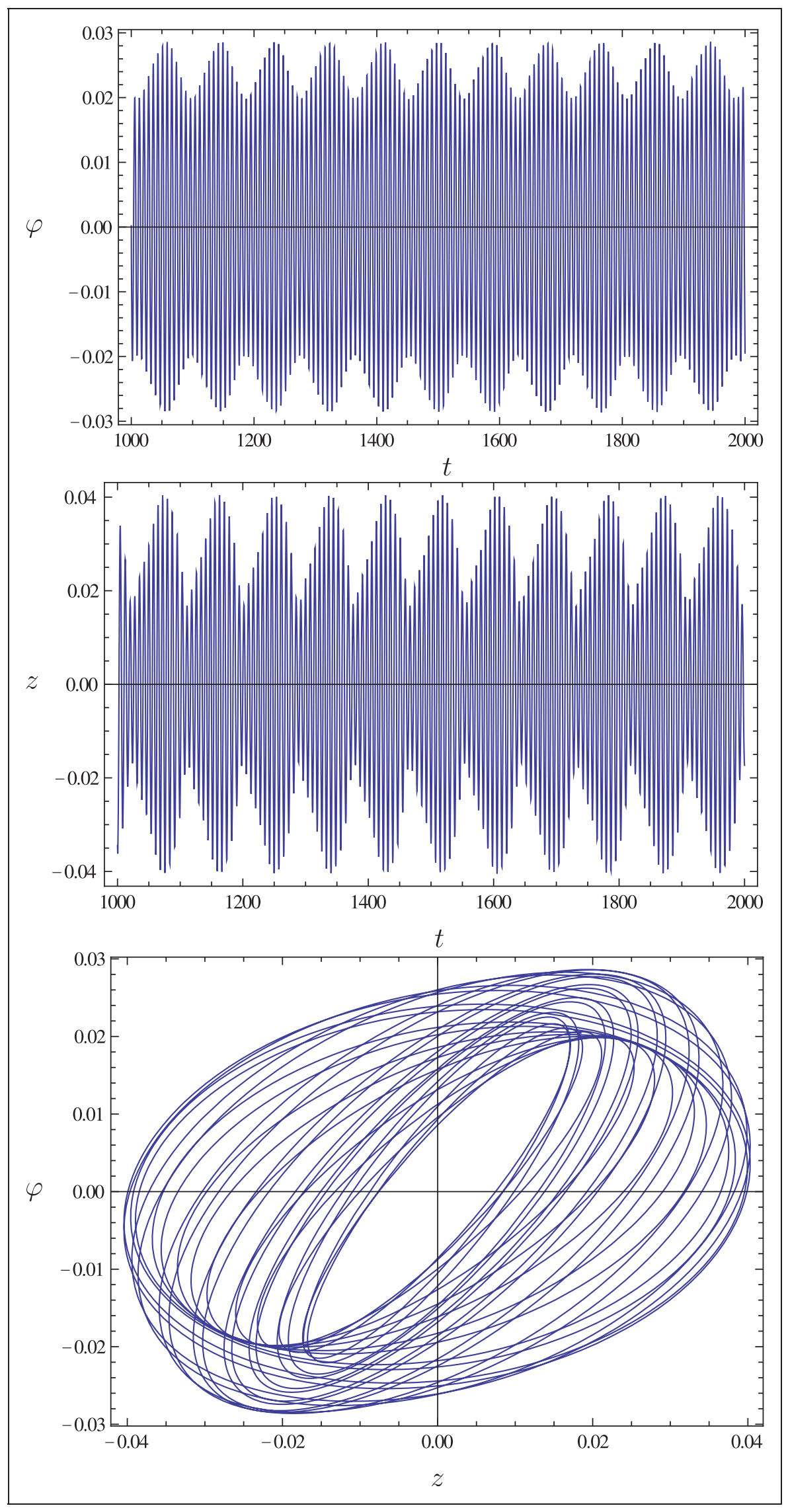

Figure 7. Numerical integration of the RAMEs, for $\kappa$

$10, \mu_{1}$

0.07 and $\delta$

0.75 . 


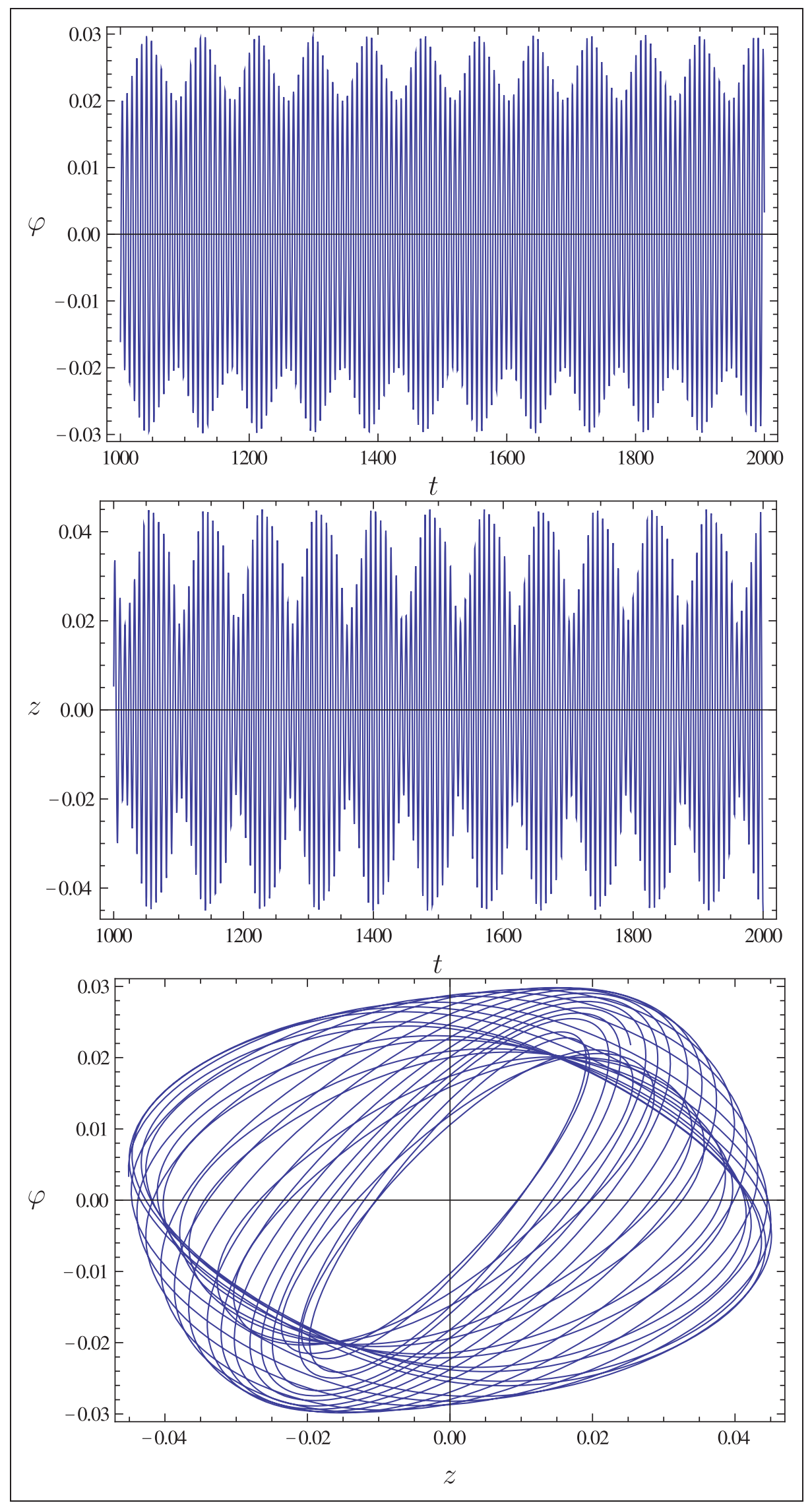

Figure 8. Numerical integration of the originating equations, for $\kappa$

10, $\mu_{1} \quad 0.07$ and $\delta$

0.75 . 
oscillations in $(x, \varphi, z)$, which are shown in Figure 7. They are in good agreement with the relevant results of the numerical integrations of the originating equations (23), shown in Figure 8.

\section{Conclusions}

In this paper, a general, nonlinear, multi-d.o.f. system, subjected to aeroelastic forces and close to a Hopf bifurcation, is considered. The system is equipped with an essentially nonlinear oscillator with small mass (Nonlinear Energy Sink, NES), with the aim of passively controlling the amplitude of vibrations of the main system. A mixed Multiple Scale/Harmonic Balance Method is proposed to get slow-flow equations, as well as the critical manifold around which the dynamics occur. The main advantage of the procedure is that no complexification is required, so that the analysis is re-conducted in the framework of the perturbation techniques. Moreover, whatever the order of the main system is, the procedure provides the Reduced Amplitude Modulation Equations, in number equal to the co-dimension three of the problem. Numerical tests on a specific example, already analyzed in Gendelman et al. (2010), constituted by a two-d.o.f. rigid wing under subsonic wind, are pursued. The results show good agreement between the outcomes of the algorithm and direct integrations of the originating equations. The presence of NES modifies the position of the bifurcation point and the amplitude of the bifurcated branch. However, as also recognized in Gendelman et al. (2010), the presence of NES in this specific system is not always beneficial, since just in case of leeward NES, the bifurcated branch is underneath the one obtained when the NES is disengaged.

\section{Funding}

This work has been supported by the Italian Ministry of Universities (MIUR) through a PRIN 20102012 program (grant number 2010MBJK5B).

\section{References}

Gendelman O (2004) Bifurcations of nonlinear normal modes of linear oscillator with strongly nonlinear damped attach ment. Nonlinear Dynamics 37: 115128.

Gendelman OV, Starosvetsky Y and Feldman M (2008) Attractors of harmonically forced linear oscillator with attached nonlinear energy sink I: Description of response regimes. Nonlinear Dynamics 51: 3146.

Gendelman O, Vakakis A, Bergman L and McFarland D (2010) Asymptotic analysis of passive nonlinear suppres sion of aeroelastic instabilities of a rigid wing in subsonic flow. SIAM Journal of Applied Mathematics 70: 16551677.
Guckenheimer J and Holmes P (1983) Nonlinear Oscillations, Dynamical Systems, and Bifurcation of Vector Fields. New York: Springer Verlag.

Guckenheimer J, Wechselberger M and Young LS (2006) Chaotic attractors of relaxation oscillators. Nonlinearity 19: 701720 .

Lamarque CH, Gendelman O, Savadkoohi A and Etcheverria E (2011) Targeted energy transfer in mechanical systems by means of non smooth nonlinear energy sink. Acta Mechanica 221: 175200.

Lee Y, Vakakis A, Bergman L, McFarland D and Kerschen G (2007) Suppressing aeroelastic instability using broad band passive targeted energy transfers, part 1: Theory. AIAA Journal 45: 693711.

Lee Y, Vakakis A, Bergman L, McFarland D and Kerschen G (2008a) Enhancing robustness of aerolastic instability suppression using mdof energy sinks. AIAA Journal 46: 13711394

Lee Y, Vakakis A, Bergman L, McFarland D and Kerschen G (2008b) Suppressing aeroelastic instability using broad band passive targeted energy transfers, part 2: Experiments. AIAA Journal 46: 23912400.

Luongo A and Zulli D (2012a) Dynamic analysis of externally excited NES controlled systems via a mixed Multiple Scale/Harmonic Balance algorithm. Nonlinear Dynamics 70(3): 20492061.

Luongo A and Zulli D (2012b) A paradigmatic system to study the transition from zero/Hopf to double zero/Hopf bifurcation. Nonlinear Dynamics 70(1): 111124.

Manevitch L (2001) The description of localized normal modes in a chain of nonlinear coupled oscillators using complex variables. Nonlinear Dynamics 25: 95109.

Musienko A, Lamarque CH and Manevitch L (2006) Design of mechanical energy pumping devices. Journal of Vibration and Control 12: 355371.

Nayfeh A (2011) The Method of Normal Forms. 2nd edn. New York: John Wiley \& Sons.

Nayfeh A and Mook D (1979) Nonlinear oscillations. New York: John Wiley \& Sons.

Pham T, Lamarque CH and Savadkoohi A (2012) Multi resonance capturing in a two degree of freedom system under two different harmonic excitations. Journal of Vibration and Control 18: 451466.

Savadkoohi A, Vaurigaud B, Lamarque $\mathrm{CH}$ and Pernot S (2012) Targeted energy transfer with parallel nonlinear energy sinks. Part II: Theory and experiments. Nonlinear Dynamics 67(1): 3746.

Starosvetsky Y and Gendelman O (2008a) Dynamics of a strongly nonlinear vibration absorber coupled to a har monically excited two degree of freedom system. Journal of Sound and Vibration 312: 234256.

Starosvetsky Y and Gendelman O (2008b) Response regimes of linear oscillator coupled to nonlinear energy sink with harmonic forcing and frequency detuning. Journal of Sound and Vibration 315: 746765.

Starosvetsky Y and Gendelman O (2009) Vibration absorp tion in systems with a nonlinear energy sink: Nonlinear damping. Journal of Sound and Vibration 324: 916939.

Starosvetsky Y and Gendelman O (2010a) Bifurcations of attractors in forced system with nonlinear energy 
sink: the effect of mass asymmetry. Nonlinear Dynamics 59: 711731 .

Starosvetsky Y and Gendelman O (2010b) Interaction of non linear energy sink with a two degrees of freedom linear system: Internal resonance. Journal of Sound and Vibration 329: 18361852.

Starosvetsky Y and Gendelman OV (2011) Response regimes in forced system with non linear energy sink: quasi periodic and random forcing. Nonlinear Dynamics 64: 177195.

Vakakis A, Gendelman O, Bergman L, McFarland D, Kerschen G and Lee Y (2008a) Nonlinear Targeted Energy Transfer in Mechanical and Structural Systems I. New York: Springer Verlag.

Vakakis A, Gendelman O, Bergman L, McFarland D, Kerschen G and Lee Y (2008b) Nonlinear Targeted Energy Transfer in Mechanical and Structural Systems II. New York: Springer Verlag.

Vaurigaud B, Manevitch L and Lamarque CH (2011) Passive control of aeroelastic instability in a long span bridge model prone to coupled flutter using targeted energy trans fer. Journal of Sound and Vibration 330: 25802595.

Vaurigaud B, Savadkoohi A and Lamarque CH (2011) Targeted energy transfer with parallel nonlinear energy sinks. Part I: Design theory and numerical results. Nonlinear Dynamics 66: 763780.

\section{Appendix A: coefficients of the equations}

The expression of the coefficients of Equation (13) is

$$
\begin{aligned}
& c_{1}=2 i \omega \mathbf{v}^{H} \mathbf{M u}+\mathbf{v}^{H} \mathbf{C}_{0} \mathbf{u} ; \quad c_{2}=-i \omega \mathbf{v}^{H} \mathbf{C}_{1} \mathbf{u}-\mathbf{v}^{H} \mathbf{K}_{1} \mathbf{u} ; \\
& c_{3}=-i \omega \mathbf{v}^{H} \mathbf{r} ; \quad c_{4}=-3 \kappa \mathbf{v}^{H} \mathbf{r} ; \quad c_{5}=-3 \mathbf{v}^{H} \mathbf{f}(\overline{\mathbf{u}}, \mathbf{u}, \mathbf{u}) ;
\end{aligned}
$$

In Equation (15) the column matrices $\mathbf{w}_{j}(j=1, \ldots, 5)$ are the solutions of the following singular algebraic problems, in which, however, compatibility is satisfied:

$$
\begin{array}{ll}
\mathbf{w}_{1}: & \left(\mathbf{K}_{0}-\omega^{2} \mathbf{M}+i \omega \mathbf{C}_{0}\right) \mathbf{w}_{1} \\
& =-\left(\mathbf{C}_{1} \mathbf{u}-\frac{1}{c_{1}}\left(\mathbf{v}^{H} \mathbf{C}_{1} \mathbf{u}\right)\left(2 i \omega \mathbf{M u}+\mathbf{C}_{0} \mathbf{u}\right)\right) \\
\mathbf{w}_{2}: & \left(\mathbf{K}_{0}-\omega^{2} \mathbf{M}+i \omega \mathbf{C}_{0}\right) \mathbf{w}_{2} \\
& =-\left(\mathbf{K}_{1} \mathbf{u}-\frac{1}{c_{1}}\left(\mathbf{v}^{H} \mathbf{K}_{1} \mathbf{u}\right)\left(2 i \omega \mathbf{M u}+\mathbf{C}_{0} \mathbf{u}\right)\right) \\
\mathbf{w}_{3}: & \left(\mathbf{K}_{0}-\omega^{2} \mathbf{M}+i \omega \mathbf{C}_{0}\right) \mathbf{w}_{3} \\
& =-\left(\mathbf{r}-\frac{1}{c_{1}}\left(\mathbf{v}^{H} \mathbf{r}\right)\left(2 i \omega \mathbf{M u}+\mathbf{C}_{0} \mathbf{u}\right)\right) \\
\mathbf{w}_{4}: & \left(\mathbf{K}_{0}-\omega^{2} \mathbf{M}+i \omega \mathbf{C}_{0}\right) \mathbf{w}_{4} \\
& =-\left(\mathbf{r}-\frac{1}{c_{1}}\left(\mathbf{v}^{H} \mathbf{r}\right)\left(2 i \omega \mathbf{M u}+\mathbf{C}_{0} \mathbf{u}\right)\right)
\end{array}
$$

$$
\begin{array}{ll}
\mathbf{w}_{5}: & \left(\mathbf{K}_{0}-\omega^{2} \mathbf{M}+i \omega \mathbf{C}_{0}\right) \mathbf{w}_{5} \\
& =-\left(\mathbf{f}(\overline{\mathbf{u}}, \mathbf{u}, \mathbf{u})-\frac{1}{c_{1}}\left(\mathbf{v}^{H} \mathbf{f}(\overline{\mathbf{u}}, \mathbf{u}, \mathbf{u})\right)\left(2 i \omega \mathbf{M u}+\mathbf{C}_{0} \mathbf{u}\right)\right)
\end{array}
$$

The solution is made unique by the normalization condition $\mathbf{w}_{j}^{T} \mathbf{u}=0$.

Moreover $\mathbf{w}_{j}(j=6,7)$ are the solutions of the following nonsingular algebraic problems:

$$
\begin{array}{ll}
\mathbf{w}_{6}: & \left(\mathbf{K}_{0}-9 \omega^{2} \mathbf{M}+3 i \omega \mathbf{C}_{0}\right) \mathbf{w}_{6}=-\mathbf{r} \\
\mathbf{w}_{7}: & \left(\mathbf{K}_{0}-9 \omega^{2} \mathbf{M}+3 i \omega \mathbf{C}_{0}\right) \mathbf{w}_{7}=-\mathbf{f}(\mathbf{u}, \mathbf{u}, \mathbf{u})
\end{array}
$$

When the right and left eigenvectors are normalized so that $c_{1}=1$, the expression of $\mathcal{F}, \mathcal{G}, \mathcal{H}$ in Equation (19) is

$$
\begin{aligned}
& \mathcal{F}=\frac{1}{4}\left(a^{3} c_{5 R}+4 a c_{2 R} \mu_{1}+b^{3} c_{4 I} \sin \gamma+b^{3} c_{4 R} \cos \gamma\right. \\
& \left.+4 b \xi c_{3 I} \sin \gamma+4 b \xi c_{3 R} \cos \gamma\right) \text {; } \\
& \mathcal{G}=-\frac{1}{4\left(4 m^{2} \omega^{2}+\xi^{2}\right)}\left(\operatorname { a m \omega } \left(a ^ { 2 } \left(( \omega ) \left(4 c_{5 I} m \mathbf{r}^{T} \mathbf{u}\right.\right.\right.\right. \\
& \left.\left.+6 m \mathbf{r}^{T} \mathbf{w}_{5 R} \omega-3 \xi \mathbf{r}^{T} \mathbf{w}_{5 I}\right)+2 c_{5 R} \xi \mathbf{r}^{T} \mathbf{u}\right) \sin \gamma \\
& +\left(2 c_{5 I} \xi \mathbf{r}^{T} \mathbf{u}+\omega\left(-4 c_{5 R} m \mathbf{r}^{T} \mathbf{u}+6 m \mathbf{r}^{T} \mathbf{w}_{5 I} \omega\right.\right. \\
& \left.\left.\left.+3 \xi \mathbf{r}^{T} \mathbf{w}_{5 R}\right)\right) \cos \gamma\right)+4\left(\left(2 c_{2 R} \mu_{1} \xi \mathbf{r}^{T} \mathbf{u}\right.\right. \\
& -\omega\left(-4 c_{2 I} \mu_{1} m \mathbf{r}^{T} \mathbf{u}+\omega\left(\mu_{1} \xi \mathbf{r}^{T} \mathbf{w}_{1 R}\right.\right. \\
& \left.-2 m\left(\mathbf{r}^{T} \mathbf{u}-\mu_{1} \mathbf{r}^{T} \mathbf{w}_{1 I} \omega+\mu_{1} \mathbf{r}^{T} \mathbf{w}_{2 R}\right)\right) \\
& \left.\left.+\mu_{1} \xi \mathbf{r}^{T} \mathbf{w}_{2 I}\right)\right) \sin \gamma+\left(2 c_{2 I} \mu_{1} \xi \mathbf{r}^{T} \mathbf{u}+\omega\left(-4 c_{2 R} \mu_{1} m \mathbf{r}^{T} \mathbf{u}\right.\right. \\
& +\mu_{1}\left(\omega\left(2 m\left(\mathbf{r}^{T} \mathbf{w}_{1 R} \omega+\mathbf{r}^{T} \mathbf{w}_{2 I}\right)-\xi \mathbf{r}^{T} \mathbf{w}_{1 I}\right)+\xi \mathbf{r}^{T} \mathbf{w}_{2 R}\right) \\
& \left.\left.\left.\left.+\xi \mathbf{r}^{T} \mathbf{u}\right)\right) \cos \gamma\right)\right)+b^{3}\left(2 m \mathbf{r}^{T} \mathbf{u} \omega\left(c_{4 I} \xi-2 c_{4 R} m \omega\right)\right. \\
& \left.+3 \kappa\left(2 m^{2} \mathbf{r}^{T} \mathbf{w}_{4 I} \omega^{3}+m \xi \mathbf{r}^{T} \mathbf{w}_{4 R} \omega^{2}+\xi\right)\right) \\
& +4 b m \omega\left(2 \xi c_{3 I} \xi \mathbf{r}^{T} \mathbf{u}+\omega\left(\xi \left(2 m \mathbf{r}^{T} \mathbf{w}_{3 R} \omega^{2}\right.\right.\right. \\
& \left.\left.\left.-\xi \mathbf{r}^{T} \mathbf{w}_{3 I}(\omega+1)-4 \xi c_{3 R} m \mathbf{r}^{T} \mathbf{u}\right)\right)\right) \text {; } \\
& \mathcal{H}=\frac{1}{4\left(4 m^{2} \omega^{2}+\xi^{2}\right)}\left(a ^ { 3 } \left(m \omega \left(2 c_{5 I} \xi \mathbf{r}^{T} \mathbf{u}+\omega\left(-4 c_{5 R} m \mathbf{r}^{T} \mathbf{u}\right.\right.\right.\right. \\
& \left.\left.+6 m \mathbf{r}^{T} \mathbf{w}_{5 I} \omega+3 \xi \mathbf{r}^{T} \mathbf{w}_{5 R}\right)\right) \sin \gamma-m \omega\left(\omega \left(4 c_{5 I} m \mathbf{r}^{T} \mathbf{u}\right.\right. \\
& \left.\left.+6 m \mathbf{r}^{T} \mathbf{w}_{5 R} \omega-3 \xi \mathbf{r}^{T} \mathbf{w}_{5 I}\right)+2 c_{5 R} \xi \mathbf{r}^{T} \mathbf{u}\right) \cos \gamma \\
& \left.+c_{5 I}\left(4 m^{2} \omega^{2}+\xi^{2}\right)\right)+4 a\left(m \omega \left(2 c_{2 I} \mu_{1} \xi \mathbf{r}^{T} \mathbf{u}\right.\right. \\
& +\omega\left(-4 c_{2 R} \mu_{1} m \mathbf{r}^{T} \mathbf{u}+\mu_{1}\left(\omega \left(2 m\left(\mathbf{r}^{T} \mathbf{w}_{1 R} \omega+\mathbf{r}^{T} \mathbf{w}_{2 I}\right)\right.\right.\right. \\
& \left.\left.\left.\left.-\xi \mathbf{r}^{T} \mathbf{w}_{1 I}\right)+\xi \mathbf{r}^{T} \mathbf{w}_{2 R}\right)+\xi \mathbf{r}^{T} \mathbf{u}\right)\right) \sin \gamma \\
& +m \omega\left(\omega \left(-4 c_{2 I} \mu_{1} m \mathbf{r}^{T} \mathbf{u}+\omega\left(\mu_{1} \xi \mathbf{r}^{T} \mathbf{w}_{1 R}\right.\right.\right. \\
& \left.\left.-2 m\left(\mathbf{r}^{T} \mathbf{u}-\mu_{1} \mathbf{r}^{T} \mathbf{w}_{1 I} \omega+\mu_{1} \mathbf{r}^{T} \mathbf{w}_{2 R}\right)\right)+\mu_{1} \xi \mathbf{r}^{T} \mathbf{w}_{2 I}\right) \\
& \left.\left.-2 c_{2 R} \mu_{1} \xi \mathbf{r}^{T} \mathbf{u}\right) \cos \gamma+c_{2 I} \mu_{1}\left(4 m^{2} \omega^{2}+\xi^{2}\right)\right)
\end{aligned}
$$




$$
\begin{aligned}
& +b\left(b ^ { 2 } \left(\left(4 m^{2} \omega^{2}+\xi^{2}\right)\left(c_{4 I} \cos \gamma-c_{4 R} \sin \gamma\right)\right.\right. \\
& -m \omega\left(2 \mathbf{r}^{T} \mathbf{u}\left(2 c_{4 I} m \omega+c_{4 R} \xi\right)+\kappa\left(6 m \mathbf{r}^{T} \mathbf{w}_{4 R} \omega^{2}\right.\right. \\
& \left.\left.\left.-3 \xi \mathbf{r}^{T} \mathbf{w}_{4 I} \omega+6\right)\right)\right)+4\left(4 m^{2} \omega^{2}+\xi^{2}\right)\left(\xi c_{3 I} \cos \gamma\right. \\
& \left.-\xi c_{3 R} \sin \gamma\right)+4 \omega\left(2 m^{2} \omega\left(\omega-2 \xi c_{3 I} \mathbf{r}^{T} \mathbf{u}\right)\right.
\end{aligned}
$$

$$
\begin{aligned}
& -2 \xi c_{3 R} m \xi \mathbf{r}^{T} \mathbf{u}+\xi\left(2 m^{2} \mathbf{r}^{T} \mathbf{w}_{3 I} \omega^{3}\right. \\
& \left.\left.\left.\left.+m \xi \mathbf{r}^{T} \mathbf{w}_{3 R} \omega^{2}+\xi\right)\right)\right)\right)
\end{aligned}
$$

where $\quad c_{j R}:=\Re\left(c_{j}\right), c_{j I}:=\Im\left(c_{j}\right), j=2, \ldots, 5$ $\mathbf{w}_{j R}:=\Re\left(\mathbf{w}_{j}\right), \mathbf{w}_{j I}:=\Im\left(\mathbf{w}_{j}\right), j=1, \ldots, 7$. 